\section{Transsartorial approach for saphenous nerve block}

Michael van der Wal MB ChB, Scott A. Lang FRCPC, Ray W. Yip FRCPC
Blockade of conduction in the saphenous nerve is important in providing surgical anaesthesia in the lower leg. Unfortunately, previously described techniques have lacked clinical effectiveness in practice. We developed a transsartorial approach for conduction block of the saphenous nerve. We first confirmed its potential clinical utility in 12 cadaveric specimens by demonstrating that the saphenous nerve was consistently stained by injections of methylene blue. Subsequently, we compared the relative rates of successful saphenous nerve block and the extent of conduction block provided by three techniques: (1) transsartorial saphenous nerve block (TSSNB), (2) above knee femoral paracondylar field block (FPFB), and (3) below knee field block (BKFB) of the saphenous nerve in 20 ASA I volunteers. The transsartorial saphenous nerve block proved to be highly successful (80\% success rate) and was superior to the other two approaches in providing cutaneous analgesia to pinprick in the saphenous nerve distribution $(P<0.05)$. The success rates of the BKFB and FPFB were $65 \%$ and $40 \%$ respectively. $A$ successful block with the transsartorial approach provided complete anaesthesia of the medial malleolus in $94 \%$ of subjects whilst the $B K F B$ and FPFB provided complete anaesthesia of the medial malleolus in less than $40 \%$ of the successful blocks. We recommend the transsartorial approach for more effective block of the saphenous nerve.

Le bloc du nerf saphène constitue une composante importante de l'anesthésie chirurgicale du membre inférieur. Malheureusement, en pratique, les techniques préconisées manquent d'efficacité. Nous avons développé une approche transmusculaire par le couturier pour le bloc du saphène. Nous avons d'abord confirmé son utilité clinique sur 12 cadavres en montrant que

\section{Key words}

ANAESTHETIC TECHNIQUE: regional;

ANAESTHETICS, LOCAL: lidocaine.

From the Department of Anaesthesia, Royal University

Hospital, University of Saskatchewan, Saskatoon,

Saskatchewan, Canada S7N $0 X 0$.

Address correspondence to: Dr. S.A. Lang, Department of Anaesthesia, Royal University Hospital, Saskatoon,

Saskatchewan, Canada S7N $0 X 0$.

Accepted for publication 30th January, 1993. le muscle couturier était régulièrement imprégné par des injections de bleu de méthylène. Par la suite, nous avons comparé chez 20 volontaires ASA I, le taux relatif de succès du bloc du nerf saphène et la qualité du bloc produit par trois techniques: 1) le bloc saphène "transcouturier 》, 2) le bloc fémoral paracondylien au-dessus du genou, 3) et le bloc par infiltration sous le genou. Le bloc transcouturier a été exécuté avec un taux de succès élevé (80\%) et nous l'avons trouvé supérieur aux deux autres techniques pour l'analgésie cutanée mesurée dans le territoire cutané du nerf saphène avec une piqûre d'épingle. Les taux de succès de l'approche haute (au-dessus du genou) et de l'approche plus basse (au-dessous du genou) ont été respectivement de 65 et $40 \%$. Le bloc transcouturier a produit une anesthésie complètement de la malléole interne chez $94 \%$ des sujets alors que les deux autres ne produisaient une anesthésie complète de la malléole interne que dans moins de $40 \%$ des cas. Nous recommandons le bloc transcouturier pour une l'anesthésie du nerf saphène.

The saphenous nerve is a pure sensory nerve which supplies sensation to the antero-medial aspect of the lower leg from the knee down to and including the medial malleolus (Figure 1). The combination of a sciatic nerve and saphenous nerve block will provide total anaesthesia of the lower leg distal to the knee.

Classically the saphenous nerve is blocked with a subcutaneous field block below the knee where the nerve emerges from behind the tendon of the sartorius muscle and lies beneath the skin (below knee field block; BKFB). ${ }^{1,2}$ An alternative approach has been described by $\mathrm{Katz}^{3}$ and Bonica, ${ }^{4}$ in which a subcutanous wheal of local anaesthetic is injected in a fan-like manner over the medial condyle of the femur (femoral paracondylar field block; FPFB). ${ }^{3,4}$

Our clinical experience with these approaches to the saphenous nerve block for surgery on the lower extremity has been unsatisfactory. This led us to develop an approach to the saphenous nerve that could achieve a conduction block of the parent trunk above the knee. We theorized that such a block would be more extensive and thus, would be more likely to be effective for surgery on the lower extremity. 


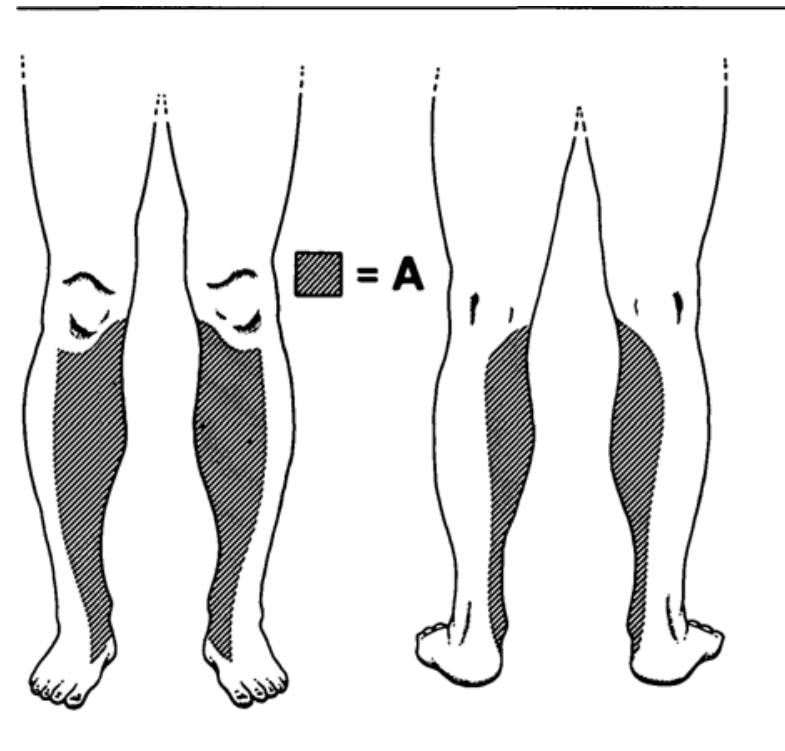

FIGURE 1 Classical cutaneous distribution of saphenous nerve (A $=$ saphenous nerve distribution).

\section{Method}

This study was performed in two stages. Ethical approval was obtained from the University of Saskatchewan's Committee on Ethics in Human Experimentation for both stages.

Stage 1 consisted of a cadaveric anatomical study, in which a transsartorial approach to the saphenous nerve was examined at a point $4 \mathrm{~cm}$ above the knee joint in 12 specimens (Figure 2). First $10 \mathrm{ml}$ methylene blue were injected into the fatpad deep to the sartorius muscle, which was identified by a loss of resistance technique, using a $4 \mathrm{~cm}$ long 20 gauge paediatric epidural Tuohy needle. This was followed by dissection of all $12 \mathrm{spec}-$ imens to determine the extent of tissue staining and spread of the injected dye as well as to review relevant anatomy. In the dissection, the sartorious muscle was exposed from the femoral triangle to its insertion below the knee (Figure 3).

Stage 2 consisted of a clinical study in 20 volunteers of ASA I status, in whom we performed and compared the two previously published approaches (FPFB and BKFB) ${ }^{1-4}$ with the transsartorial block of the saphenous nerve (TSSNB). The subjects were all fasted and $i v$ access was established before performance of the blocks. To control for individual variability, subjects were used as their own controls as all three blocks were performed in each subject. The three blocks were performed over two days in randomized order. On the first day the first two blocks were performed 30-45 min apart. Only one block was performed in each leg, using different approaches. The

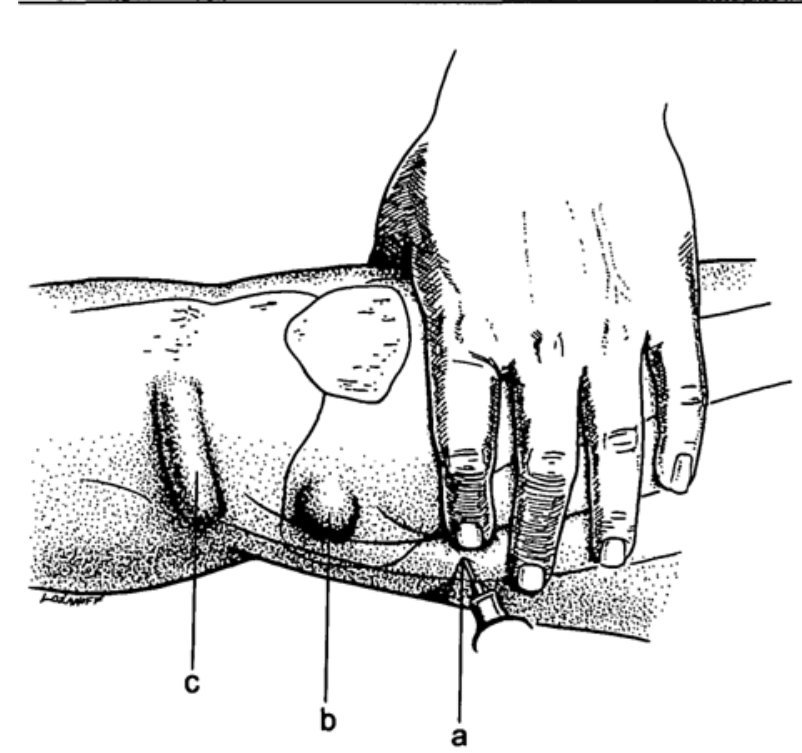

FIGURE 2 Three approaches to saphenous nerve conduction block. (Antero-medial view of right leg; (a) Transsartorial approach to the saphenous nerve; (b) Femoral para-condylar approach to the saphenous nerve; ${ }^{3,4}$ (c) Below-knee field block. ${ }^{1,2}$ )

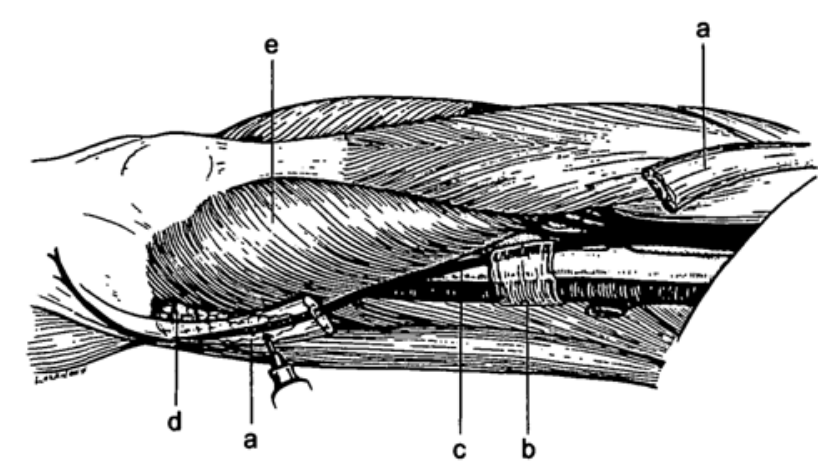

FIGURE 3 The saphenous nerve and relevant anatomy. Diagramatic depiction of the cadaveric dissections. (Antero-medial view of right leg; (a) sartorious muscle; (b) fascial envelope containing saphenous nerve, superficial femoral artery (white) and femoral vein (black-white stripes); (c) saphenous nerve block; (d) fat pad beneath sartorius muscle within which lies the saphenous nerve; (e) vastus medialis).

next day the third block was performed after confirming the return of normal sensation. Each block was assessed by one of the investigators and by an independent observer. All blocks were tested by pinprick. Testing was done every $60 \mathrm{sec}$ until onset. "Onset" was defined as the time elapsed from needle insertion until loss of pinprick sensation as first noted at a point more than 5 
$\mathrm{cm}$ distal to the site of needle insertion. The full extent of anaesthesia was assessed $30 \mathrm{~min}$ after the performance of the block. A "successful block" was defined as loss of sensation to pinprick distal to the midpoint between the knee joint and the medial malleolus. The "duration" of the block was defined as the time from completion of the injection until the subject could again detect normal sensation to pinprick $5 \mathrm{~cm}$ distal to the needle insertion site. Subjects were asked to test their own blocks hourly until resolution.

The transsartorial block (Group A) was performed with a 20 gauge Tuohy pediatric epidural needle. In the supine subject a skin wheal of local anaesthetic was raised with a 27 gauge needle over the sartorial muscle belly. The sartorius muscle is easily palpated on the medial side, just above the knee, when the extended leg is actively elevated one or two inches. The level at which the needle was introduced was located by drawing a perpendicular (vertical) line down from a point, one finger width above the patella in the supine extended leg. The Tuohy needle was then advanced slightly posteriorly $\left(45^{\circ}\right)$ from the coronal plane and slightly caudad, through the muscle belly of sartorius until a loss of resistance identified the subsartorial fatpad (Figure 2). This loss of resistance occurred at a depth ranging from $1.5-3.0 \mathrm{~cm}$ depending on the size and body habitus of the subject. Following careful aspiration, $10 \mathrm{ml}$ lidocaine $2 \%$ with epinephrine 1:200,000 were deposited.

The para-condylar block (Group B) was performed exactly according to the description by Katz ${ }^{3}$ and Bonica. ${ }^{4}$ A skin wheal of local anaesthetic was raised with a 27 gauge needle over the bony prominence of the medial femoral condyle. With a 22 gauge B-bevel needle, $6 \mathrm{ml}$ lidocaine $2 \%$ (as per the original description by Katz) ${ }^{3}$ with 1:200,000 epinephrine were deposited in a fan-like manner between the skin and periosteum of the medial surface of the medial femoral condyle (Figure 2).

The classical below-knee block (Group C) was done with $10 \mathrm{ml}$ of lidocaine $2 \%$ with epinephrine $1: 200,000$ via a 22 gauge needle, through a skin wheal of lidocaine made with a 27 gauge needle. A linear subcutaneous deposition of local anaesthetic, $5 \mathrm{~cm}$ long, was made immediately below the insertion of the sartorius tendon at the tibial tubercle in an anterolateral-medial plane (Figure 2).

The extent of the successful blocks was recorded on three view diagrams of the leg. All complications, immediate and delayed ( $48 \mathrm{hr}$ ), were recorded.

Statistical comparisons among the groups were analyzed using the two-tailed Fisher exact test and the Wilcoxon signed rank test, where appropriate, with $P<$ 0.05 indicating statistical significance.
TABLE I Demographic data of 20 ASA I volunteers

\begin{tabular}{ll}
\hline & Mean values \pm SD \\
\hline Age (yr) & $31.6 \pm 3.75$ \\
Sex (M:F) & $4: 1$ \\
Weight $(\mathrm{kg})$ & $72.2 \pm 10.2$ \\
Height $(\mathrm{cm})$ & $173.8 \pm 9.0$ \\
\hline
\end{tabular}

\section{Results}

\section{Stage 1}

The saphenous nerve was found to travel with the femoral artery and vein, within the neurovascular sheath, from the femoral triangle as far as the adductor hiatus. At this point the superficial femoral artery and vein passed through the adductor hiatus, into the popliteal fossa, whilst the saphenous nerve continued on its original course just underneath the sartorius muscle. From the adductor hiatus, the saphenous nerve travelled within the fascia/fat layer overlying the tendon of the adductor magnus, for a distance varying between $3-8 \mathrm{~cm}$. The saphenous nerve could consistently be found in this inter-fascial plane (Figure 3). Also, the methylene blue was consistently found to spread in this plane and staining of the saphenous nerve was evident in all the specimens.

\section{Stage 2}

Demographic data for the volunteers are in Table I.

The overall success rate of the transsartorial block of the saphenous nerve in our volunteer group was $80 \%$ $(16 / 20)$. This was not different from the below-knee field block success rate of $65 \%(13 / 20)$ but it was better than the $40 \%(8 / 20)$ success rate achieved with the paracondylar approach described by $\operatorname{Katz}^{3}(P<0.05$; Table II).

There was a difference in the rate with which "successful" blocks achieved complete anaesthesia of the medial malleolus via the transsartorial approach $(94 \%)$ when compared with the below-knee approach $(39 \%)(P=$ $0.001)$ and the para-condylar approach $(25 \%)(P<0.01$ : Figure 3; Table II). With the exception of one patient in the below-knee group, analgesia above the knee joint was found only in the transsartorial group.

The times of onset and durations of action of the blocks were similar for the three different approaches (Table III).

There were no serious complications in any of the 20 volunteers following any of the blocks. Minor complications, such as bruising and tenderness at the injection site, occurred with the same frequency with all three approaches. One individual experienced a short-lived partial 
TABLE II Success rates and extent of saphenous nerve blocks

\begin{tabular}{llll}
\hline & \multicolumn{2}{l}{ Route of block } & \\
\cline { 2 - 4 } & $\begin{array}{l}\text { TSSNB } \\
(n=20)\end{array}$ & $\begin{array}{l}\text { FPFB } \\
(n=20)\end{array}$ & $\begin{array}{l}\text { BKFB } \\
(n=20)\end{array}$ \\
\hline $\begin{array}{l}\text { No of successful blocks } \\
\begin{array}{l}\text { No. of successful blocks } \\
\text { extending to medial } \\
\text { malleolus }\end{array}\end{array}$ & $16(80 \%)^{*} \ddagger$ & $8(40 \%) \S$ & $13(65 \%)$ \\
\hline
\end{tabular}

TSSNB $=$ Transsartorial saphenous nerve block.

FPFB = Femoral para-condylar field block.

$\mathrm{BKFB}=$ Below knee field block.

$P<0.05:$ * vs FPFB; $\uparrow$ vs BKFB; Nonsignificant: $\ddagger$ vs BKFB; $\S$ vs BKFB.

TABLE III Onset and duration of successful blocks

\begin{tabular}{llll}
\hline & $\begin{array}{l}\text { TSSNB } \\
(n=16)\end{array}$ & $\begin{array}{l}\text { FPFB } \\
(n=9)\end{array}$ & $\begin{array}{l}B K F B \\
(n=13)\end{array}$ \\
\hline Onset (min) & $5.1 \pm 2.9$ & $7.7 \pm 5.6$ & $8.3 \pm 5.6$ \\
Duration (hr) & $6.4 \pm 1.9$ & $5.8 \pm 2.9$ & $5.8 \pm 2.6$ \\
\hline
\end{tabular}

TSSNB $=$ Transsartorial saphenous nerve block.

FPFB $=$ Femoral para-condylar field block.

$\mathrm{BKFB}=$ Below knee field block.

(All comparisons statistically insignificant.)

femoral nerve palsy characterized by analgesia to pinprick over the anterior thigh and minor weakness of the quadriceps muscle after the transsartorial block. Three subjects reported brief paraesthesia in the distribution of the saphenous nerve during needle insertion with the transsartorial technique. There were no neurological complications. Four subjects had mild systemic symptoms of local anaesthetic toxicity (lightheadedness, tinnitus) after completion of the two successive saphenous nerve blocks performed on the first day.

\section{Discussion}

To provide successful peripheral regional anaesthesia for surgery involving the lower leg or ankle, cutaneous analgesia in the distribution of the saphenous nerve must be achieved. ${ }^{5}$ This can be produced by blocking either the femoral or saphenous nerve. The advantage of the saphenous nerve block is that there is no loss of motor power. Although several approaches to the saphenous nerve have been described, ${ }^{1-4}$ to our knowledge no studies have been published that document or compare the success rates. We have described a new transsartorial approach to the saphenous nerve based upon anatomical dissection.

The success rates of this new approach and two pre- viously described approaches, as well as the extent of the blocks achieved with each approach, were compared.

The highest rate of successful blocks was achieved with the transsartorial approach $(80 \%)$. The statistically insignificant difference in success between the transsartorial $(80 \%)$ and the below-knee $(65 \%)$ methods likely represents insufficient power (20\%) of our study to demonstrate a difference of this magnitude rather than a true lack of a clinical difference. Approximately 150 patients would have been required to achieve an $80 \%$ likelihood of demonstrating this difference statistically (that is, a $15 \%$ difference at a two-tailed alpha of 0.05 ). ${ }^{6}$

A higher rate of complete anaesthesia of the medial malleolus could be achieved using the transsartorial approach (94\%) than with the below-knee $(39 \% ; P=0.001)$ and the para-condylar approaches $(25 \% ; P<0.01)$. Many of the blocks performed by the previously described two methods (BKFB and FPFB) had to be considered "successful" in that they provided anaesthesia in approximately $80 \%$ of the area supplied by the saphenous nerve, but still did not provide anaesthesia over the medial malleolus. Clinically this would be important in that the blocks would not have provided adequate anaesthesia for ankle surgery. We therefore documented two endpoints: (1) rate of successful blocks and (2) the presence of anaesthesia over the medial malleolus.

Although the saphenous nerve can be blocked anywhere along its course in the leg, " we feel that the transsartorial approach is the method of choice. This approach employs a loss of resistance technique with which most anaesthetists are familiar. The method also provides a definite end-point for needle and local anaesthetic placement as opposed to field block methods. There is little risk of vascular injury or intravascular injection with the transsartorial approach as the superficial femoral vessels and the saphenous nerve part company at the adductor hiatus, which lies well above our recommended site of injection. Finally, the greater distal spread of anaesthesia with the transsartorial approach predicts a higher success rate in achieving complete anaesthesia over the medial malleolus. This observed difference could be explained by the fact that the transsartorial approach achieves a block of the parent trunk at a more proximal point in its course, proximal to the origins of any terminal branches. One possible criticism of our methodology is that the results may have been more comparable if all three approaches to the saphenous nerve block were performed in the same leg of each volunteer. In the interest of time it was decided to use both legs in each subject, but individual variations in the anatomy and distribution of the saphenous nerve do exist. However, these variations are always equally present in both legs of each indivi- 
dual ${ }^{8}$ We thus felt comfortable that the utilization of both legs would not invalidate our findings. The clinical utility of this newly described approach to the saphenous nerve block is undergoing investigation.

\section{Acknowledgements}

The authors wish to thank all the volunteers, the Department of Anatomy (University of Saskatchewan), our anatomical illustrator (Beth Lozanoff), and our secretaries (Department of Anaesthesia, Royal University Hospital, Saskatoon, Saskatchewan) for their help in completing this project and in the preparation of the manuscript.

\section{References}

1 Kofoed $\mathrm{H}$. Peripheral nerve blocks at the knee and ankle in operations for common foot disorders. Clin Orthop 1982; 168: 97-101.

2 Miller $R D(E d$.). Anesthesia, Vol 2, 2nd ed. New York: Churchill Livingstone Inc., 1986; 1039-40.

3 Katz J. Atlas of Regional Anesthesia. Norwalk, Connecticut: Appleton-Century-Crofts, Prentice-Hall Inc., 1985: 156-7.

4 Bonica JJ. The Management of Pain, Vol 1, 2nd ed. Philadelphia: Lea \& Febiger, 1990: 1928.

5 Bridenbaugh $P O$. The lower extremity: somatic blockade. In: Cousins MJ, Bridenbaugh PO (Eds.). Neural Blockade in Clinical Anesthesia and Management of Pain, 2nd ed. Philadelphia: J.B. Lippincott Co., 1988: 417-41.

6 Cohen J. Statistical Power Analysis for the Behavioral Sciences. 2nd ed. Hillsdale, NJ: L. Erlbaum Associates, 1988; 195.

7 Romanoff ME, Cory PC Jr., Kalenak A, Keyser GC, Marshall $W K$. Saphenous nerve entrapment at the adductor canal. Amer J Sports Med 1989; 17: 478-81.

8 Hunter LY, Louis DS, Ricciardi JR, O'Connor GA. The saphenous nerve: its course and importance in medial arthrotomy. Amer J Sports Med 1979; 17: 227-30. 